\title{
Enseignement/apprentissage des sports à connotation masculine à l'école Tunisienne : étude des révélations des filles lors d'un cycle de football
}

\author{
Maher Guerchi ${ }^{1}$, Hajer Sahli ${ }^{2}$, Mohamed Ali Hammami ${ }^{2}$, Makrem Zguibi ${ }^{2}$ \\ ${ }^{1}$ EFTS - Laboratoire Education, Formation, Travail et Savoirs UMR - Université de Toulouse, France \\ ${ }^{2}$ Institut supérieure du sport et d'éducation physique du Kef, Tunisie
}

\begin{abstract}
Résumé : Notre recherche exploratoire s'intéresse à l'étude des révélations des élèves filles dans l'enseignement- apprentissage d'un sport à connotation masculine. Nous mettons l'accent sur les perceptions évoquées par les filles lors des interactions didactiques effectives avec l'enseignant. À l'aide de la technique des incidents critiques, (86 filles) de 7ème, 8ème et 9ème année de base ont été sollicitées à l'aide d'un questionnaire pour citer des situations injustes vécues lors d'un cycle de football avec leur enseignant. Les situations d'injustices citées par les filles $(N=312)$ ont fait l'objet d'une analyse de contenu à modèle ouvert (L'Écuyer, 1988). Cette analyse a permis l'émergence de plusieurs catégories d'injustices vécues par les filles. Ces catégories constituent une source d'information de première importance pour les enseignants d'EPS désireux d'offrir à leurs élèves un environnement éducatif mieux adapté à leurs besoins.
\end{abstract}

\section{Introduction}

Le processus d'enseignement-apprentissage est déterminé à la foi par l'enseignant et l'apprenant dans un contexte éducatif (Dyson, 1995). Selon ce postula, nombreux recherches s'intéressent d'avantage à la place qu'elle occupe l'apprenant aux seins de ce système. Ces études accordent un intérêt particulier pour l'étude des perceptions des élèves (Martinek, 1988), (Dyson, 1995), (Gagnon, Martel, Brunelle, Tousignant, Spallanzani, 2006) et récemment les travaux de (Vanoutrive, Friant, Derobertmasure, 2011). Dans le domaine des sciences de l'éducation on trouve plusieurs recherches sur les sentiments d'injustice des élèves (Meuret, 1999) mais dans le secteur de l'éducation physique ils sont très rares, comme l'étude canadienne de (Martel \& al, 1999).

Les sentiments d'injustice sont présents dans la société, à l'école et plus particulièrement en Éducation Physique et Sportive. Dans une séance d'EPS les contenus d'enseignement-apprentissage sont enseignés et évalués au masculin, la planification des activités connotées «masculines », des pratiques «étrangères » pour les filles, des barèmes peu sensibles aux différences génétiques (Cleuziou, 2000; Combaz, 1992). Les interactions enseignant-apprenants se caractérisent par les inégalités de traitement, elles sont souvent en faveur des garçons (Couchot-schiex \& Trottin, 2005), l'orientation masculine de l'EPS permet la réussite des garçons que celle de filles (Combaz, 1992 ; Cleuziou, 2000 ; Vigneron, 2005). Ces inégalités entre les filles et les garçons accentuent le sentiment d'injustices chez les élèves filles. En milieu scolaire (Debardieux, 1999 ; Dubet, 1999 ; Merle, 1999 ; Rayou, 1999) les sentiments d'injustices sont constatés le plus souvent au plan de l'évaluation, des décisions d'orientations, de la relation pédagogique et des punitions.

Pour étudier le sentiment d'injustices, les recherches accordent un intérêt particulier aux perceptions des élèves durant la séance, puisque ces derniers influencent implicitement ou explicitement leurs apprentissages. Dans la même ligne des idées, Piéron, Delfosse, Ledent et Cloes (2000), signalent que l'avis des élèves sur les situations vécues en classe permet de comprendre la signification des comportements qu'ils adoptent. Souvent ces perceptions et ces révélations des apprenant ont mit l'accent sur les situations d'injustices vécues en milieu scolaire. Plusieurs recherches ont démontré que les situations d'injustices sont fréquentes sur les terrains et qui ont des effets néfastes sur le climat de la classe d'une part et sur l'apprentissage des élèves d'autre part. Nous prenons l'exemple de la planification des activités qui favorise davantage la participation des garçons que des filles (Scraton, 1992; Rail et Dallaire, 1997). D’une autre coté, les élèves faibles reçoivent moins d'encouragements (Martinek, 1988; Martinek et Karper, 1986), bénéficient de moins d'occasions de participation dans la construction de projets d'action et de répondre aux questions de l'éducateur physique. Ils ont peu d'interaction avec leur enseignant d'éducation physique et reçoivent moins d'information sur les comportements moteurs attendus (Brown, 1979; Martinek, 1989 ; Martinek et Karper, 1986).

En milieu scolaire, il est important de prendre au sérieux ces perceptions. Or les situations d'injustice répétées " attaquent la relation pédagogique en son cour » (Desvignes \& Meuret, 2009, p.188), ou encore accompagnent la colère, la violence, la délinquance (Bègue, 2009).

Les perceptions et les déclarations des élèves (filles ou garçons) relativement aux injustices vécues en éducation physique constituent une source d'information de première importance pour les enseignants afin d'adapter son 
enseignement aux attentes de ces élèves que ce soit filles ou garçons et elles sont d'une grande importance pour le processus de régulation de l'enseignement.

Notre recherche s'intéresse d'avantage à l'étude des perceptions des élèves filles appartenant à des écoles Tunisienne durant un cycle de football ; un sport à connotation masculine.

\section{Matériel et Méthodes}

\section{a. Population}

Notre échantillon est composé des élèves d'une classe de $8^{\text {ème }}, 9^{\text {ème }}$ année de base et $1^{\text {ère }}$ année secondaire dont 86 filles (1'âge moyen des filles est de 13.7 ans) ont été invitées à citer des situations perçues comme des situations injustes lors d'un cycle de football avec leur enseignant.

Tableau 1. Nombre des filles selon l'établissement scolaire

\begin{tabular}{|l|c|c|}
\hline Etablissements & Filles & $(\%)$ \\
\hline Collège & 63 & $66 \%$ \\
\hline Lycée & 23 & $34 \%$ \\
\hline Total & 86 & $100 \%$ \\
\hline
\end{tabular}

\section{b. Procédure expérimentale}

A l'aide de la technique de prélèvement des incidents critiques (Brunelle, Drouin, Godbout et Tousignant, 1988) on a essayé de recueillir les situations d'injustices qui interpellaient le plus les filles durant une situation d'enseignement-apprentissage. La passation des questionnaires se réalise en l'absence de l'enseignant pour que les filles puissent s'exprimer librement.

Elles devaient compléter, individuellement un questionnaire avec la langue arabe et la langue française, avec des questions ouvertes parmi ces questions nous citons « Raconte une situation qui s'est produite durant le cycle de football que tu as trouvé injuste pour toi ou d'autres filles».

$$
\text { "عند ممارسة كرة القدم، اذكري وضعية عثنها تعتبرين فيها ان الأ ستاذ قد اخطأ في حقك اوفي حقلك تلميذة اخرى؟" }
$$

Pour répondre les filles ont été libres d'écrire en langue arabe ou française.

\section{c. Analyse des questionnaires}

Pour l'analyse des questionnaires on a opté à deux types d'analyse : une analyse quantitatif et analyse qualitatif, la dernière porte sur l'analyse du contenu des questions ouvertes.

De point de vue quantitatif, les situations d'injustices citées par les filles sont au nombre de (312 situations). Les questions ouvertes ont fait l'objet d'une analyse de contenu selon le modèle de (L'Écuyer, 1988). Il n'existe pas de catégorie au départ, ces catégories sont identifiées au fur et à mesure de l'analyse du contenu des questions ouvertes. Les situations d'injustices identifiées sont regroupées selon des catégories en se basant sur leurs similitudes. Afin de regrouper celles qui permettant ainsi l'émergence d'une typologie qui identifie les catégories d'injustices vécues par les filles nous se référons dans ce travail à la typologie proposées par (Gagnon, Martel, Brunelle, Tousignant, Spallanzani, 2006) qui regroupe les catégories d'injustices en six familles (Gestion de la discipline, Activités d'apprentissage, Gestion du temps, Préférences de l'enseignant, Comportements de pairs, Jugement de l'enseignant). Sur la base des perceptions des élèves filles, nous avons regroupé les différentes catégories d'injustices en quatre familles d'injustices (Gestion de la discipline, Activités d'apprentissage, Gestion du temps, Préférences de l'enseignant)

Dans la présente recherche, nous présentons l'exemple de la «Gestion de la discipline» puisque nous le considérons comme un model représentatif de l'injustice vécu par les filles durant un cycle de football à l'école Tunisienne. Suite à l'analyse du contenu des questions ouvertes, on pu identifier plusieurs catégories comme « Insultassions verbales », « Sanctions non verbales », « Mauvaises réactions gestuelle de l'enseignant », « Mauvaises application de règles de conduite » et «Ignorer volontairement la participation d'une fille ». Ces catégories ont été regroupées dans la famille d'injustices «Gestion de la discipline » Gagon et al (2006).

\section{Résultats}

Les résultats obtenus révèlent que les filles signalent surtout des injustices associées à la "gestion de la discipline ». Plus spécifiquement, $49 \%$ des situations injustes rapportées par les filles mettent en cause la façon dont leur enseignant d'EPS gère la discipline au sein de la classe.

L'analyse quantitative, montre que les incidents vécus par les filles sont réparties selon quatre catégories d'injustices. Pour la catégorie "gestion de la discipline» on note 153 incidents (49\%), pour l'activité d'apprentissage 57 incidents (18\%), pour la gestion du temps 68 incidents $(22 \%)$ pour les préférences de l'enseignant 34 incidents (11\%). Ces propos sont illustrés dans la figure suivant. 


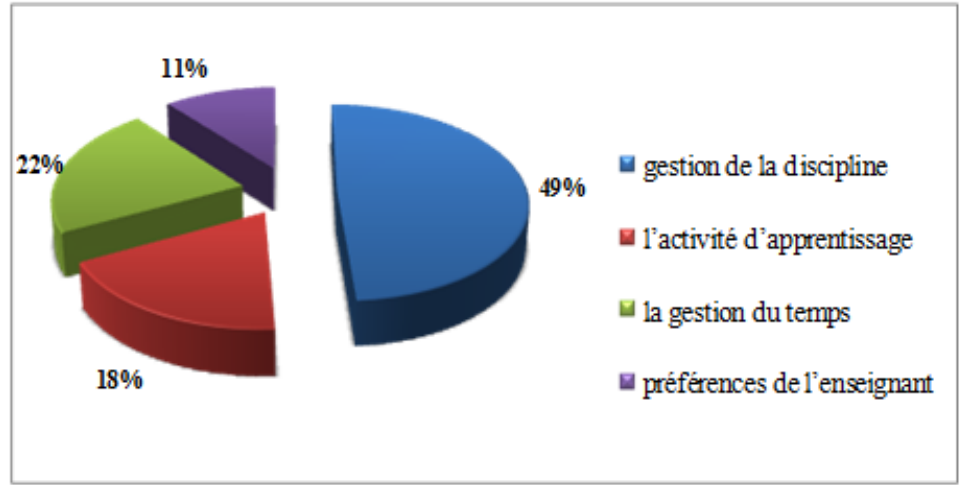

Figure 1. Répartation des situations racontées par les filles en fonction des familles d'injuctices

\section{a. Injustices liées à la gestion de la discipline}

Notre analyse révèle que l'injustice liée à la gestion de la discipline est réparti en cinq catégories avec des sous catégories. Ces catégories sont présentées dans le tableau suivant.

Tableau 2. Injustices liées à la gestion de la discipline

\begin{tabular}{|l|c|c|}
\hline \multicolumn{1}{|c|}{ Catégories d'injustices (la gestion de la discipline) } & $\begin{array}{c}\text { Nombre des situations } \\
\text { d'injustices }\end{array}$ & $\mathbf{( \% )}$ \\
\hline 1. Insultassions verbales & $\mathbf{5 0}$ & $\mathbf{3 5 \%}$ \\
\hline 2. Sanctions non verbales & $\mathbf{3 9}$ & $\mathbf{2 7 \%}$ \\
a) Faire des tours de piste & 17 & $44 \%$ \\
b) Faire des flexions/saut en extensions & 14 & $36 \%$ \\
c) faires des pompes & 08 & $20 \%$ \\
\hline 3. Mauvaises réactions gestuelles de l'enseignant & $\mathbf{2 5}$ & $\mathbf{1 7 \%}$ \\
a) Des grimasses lorsqu'elles ne comprennent pas & 11 & $44 \%$ \\
b) Des cris gênantes durant l'exercice & 14 & $56 \%$ \\
\hline 4. Mauvaises application de règles de conduite & $\mathbf{2 0}$ & $\mathbf{1 4 \%}$ \\
\hline 5. Ignorer volontairement la participation d'une fille & $\mathbf{1 0}$ & $\mathbf{0 7 \%}$ \\
\hline
\end{tabular}

Durant les séances de football, les filles ont cité 39 situations où elles jugent que leur enseignant été trop sévère avec eux. Cette catégorie d'injustice regroupe des sous catégories de la sanction proposée par l'enseignant. Nous citons $(44 \%)$ des sanctions sous forme des «tours de piste », (36\%) de ces sanctions sont en forme de «flexion saut en extension» et seulement (20\%) de ces sanctions consiste à faire des «pompes». Cette catégorie d'injustice révèle en quelque sorte que plusieurs filles pensent que leur enseignant se montre trop sévère et abuse de son autorité face à un comportement perturbateur. Les révélations qui suivent illustrent cette perception des filles « Un jour, l'enseignant $(X)$ avait dit de ne pas jouer avec les ballons au moment de l'explication de la situation, mais un de mes amies a échappé involontairement son ballon. Alors, il lui a dit d'aller faire 5tours de pistes...»; Une autre fille explique : "Une fois, j'ai parlé avec mon ami durant le déroulement de la tache, alors mon enseignant m'a dit de faire dix pompes. J'ai trouvé cela injuste...».

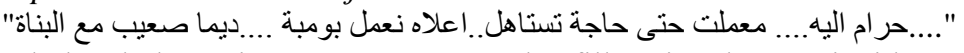

D'autres filles contestent les insultassions verbales de l'enseignant. A ce propos les filles signalent 50 incidents vécues durant le cycle de football, lorsqu'elles ne comprennent pas ses instructions « Moi et d'autres filles, nous étions en train de jouer le football, une activité que l'on n'avait jamais joué auparavant. Quand j'ai raté la balle, l'enseignant m'a crié après. Et il m'a dit que je joue comme veille..... C'est arrivé à d'autres filles. Cela me met en colère et je n'ai plus le goût de jouer encore le football...»;

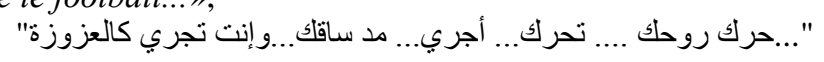

Dans le même sens une autre fille ajoute "Une fois, nous faisions un exercice de criss-cross avec la balle et une fille n'a pas été capable de le réussir. L'enseignant l'a insulté on disant Merde. Cela me fait de la peine ..., je n'aimerais pas qu'on me dise des gros mots... »

و يقول ميردة.... كلنا علاها مرة نحن نلعب بالكرة... كانت وظعية صعيبة على البنات.... صحبتي منجمتش تتحكم ف الكرة... و الأستاذ ول يصيح

D'autres révélations mettent l'accent sur les réactions gestuelles de l'enseignant. À cet égard, 25 incidents racontés par des filles durant laquelle elles ont observé des comportements gestuelles suite à une mauvaise exécution de la tache demandée. Ses réactions gestuelles se manifestent sous forme soit des grimasses avec un pourcentage de (44\%), soit sous forme des cris gênantes durant l'exercice avec un pourcentage de (56\%). Une fille raconte que «une fois l'enseignant ma demandé de faire un marquage stricte pour un garçon ... mais malheureusement je n'arrive pas à gérer le rythme du garçon ...alors j'ai resté dans un coin du terrain et 
l'enseignant se met à crier ... » elle ajoute «lorsque j'ai essayé de l'expliquer que je ne peux pas jouer avec un garçon... alors il se met à tourner la tête... ».

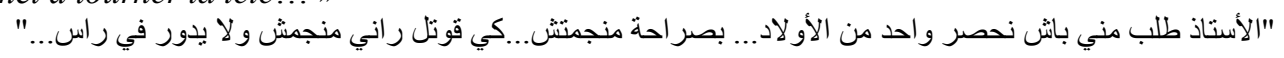

Les filles contestent aussi les punitions jugées non méritées. Certaines filles n'ont pas compris pourquoi elles subissent des punitions de groupe lorsqu'elles n'ont rien faites. A cet égard, nous présentons quelques révélations représentatives d'une injustice cité par l'une des filles «nous étions entrains de faire l'échauffement et quelqu'un avaient mal agi...il a prononcé un gros mot ...alors l'enseignant a exigé 30 minute de course pour toute la classe...alors je me demande pourquoi il punit tout le monde....».

$$
\text { "مرة ثمة ولا لعب بالأدو اة الي حتهم الأستاذ ...وقت جاء الأستاذ و مالقهش في بقعتهم عاقب الناس الكل... ". }
$$

En plus, des filles sont exaspérées lorsqu'elles considèrent, comme dans les exemples qui suivent, que leur enseignant se trompe du responsable lorsqu'il donne une punition "Olfa m'a frappée avec la balles. Mon enseignant pensait que je jouais avec les balles et il m'a mis moins un. J'ai trouvé cela injuste et je n'ai pas aimé cela...»; « une fois on jouant au football, l'enseignant $(X)$ a averti une fille qui n'avait rien fait, car c'était un autre élève qui avait frappé le ballon avec le pied. Je trouve cela injuste...».

Par ailleurs, selon les histoires des filles, pas mal d'enseignants semblent parfois Ignorer volontairement les filles. A ce sujet nous retenons 10 situations d'injustices en rapport avec cette catégorie d'injustices, les révélations suivante indiquent ce propos "une fois l'enseignant demande qui veut faire la démonstration de frapper la balle avec le cou de pied... alors j'ai voulu faire la démonstration mais l'enseignant a fait semblance de m'ignorer .... ».

"طلب منا الأستاذ شكون ورينا كيفاش نشوتوا الكورة ...حبيت نجرب... لكن الأستاذ عمل روح ما شافيش... وطلب من ولا باش ورينا... " En fait, ces révélations reflètent les sentiments d'injustice dans les relations vécues entre les filles et leurs enseignants durant un cycle de football planifié à l'école Tunisienne. Souvent ces sentiments d'injustice sont ressentis face à des situations concrètes, et non par rapport à un idéal absolu (Caillet, 2009). Ainsi, Desvignes et Meuret (2009), considèrent le sentiment d'injustice comme « un écart entre la conception de la justice des enseignants et celle des élèves»(p. 192). Il nous semble que le respect, surtout dans les témoignages d'injustices vécues par les filles, entre souvent en jeu. Desvignes et Meuret (2009) constatent que "le respect contribue davantage au sentiment général de justice que les deux autres critères, proches l'un de l'autre » (p. 194). Lors de la pratique du football, les filles ressentent les manquements au respect de leur personne, ce qui détermine plus fortement leurs sentiments d'injustice, ce que Dubet (2004) constate également par leur intensité. Ainsi, la figure suivant illustre notre propos concernant la gestion de la discipline du football.

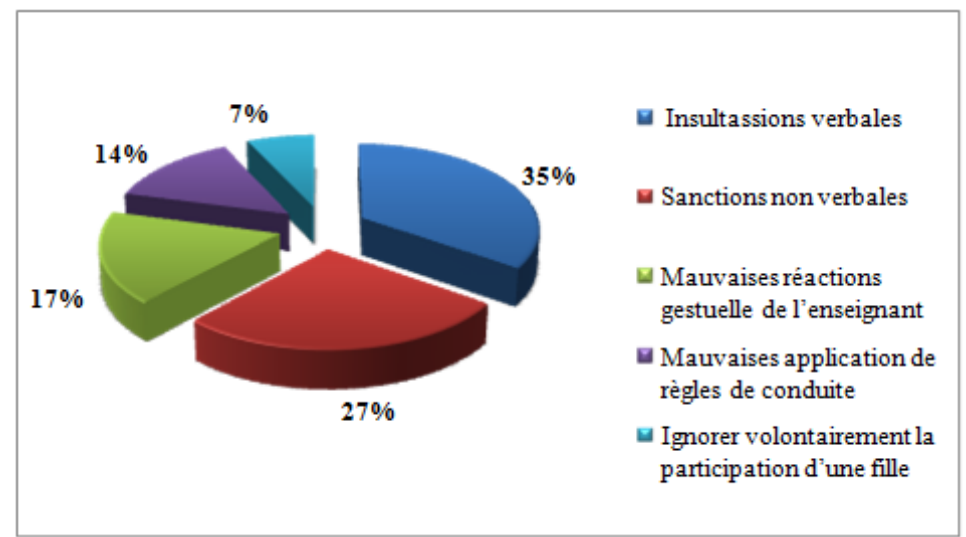

Figure 2. Catégorie d'injustices concernant (la gestion de la discipline)

\section{Discussion}

L'objectif de notre étude était l'étude des perceptions des filles concernent les situations injustes qu'ils estiment vivre durant les séances de football. Les filles perçoivent des injustes à l'égard des conditions d'apprentissage et a propos les interactions enseignants-apprenant. Certaines filles signalent que leur enseignant recours souvent à la punition sans justification et il manque de constance dans l'application des sanctions. En fait, l'utilisation répétitive d'interventions autoritaires risque de créer, chez les filles, un état de conformisme qui les amène à se soumettre et à vivre silencieusement une violence intérieure (Artaud, 1989 ; Debarbieux, 1990). A engendrer une élévation de comportements déviants chez d'autres élèves. En effet pour réduire les risques d'un conformisme dans lequel peuvent se réfugier les filles ou les garçons, Dostie (1996) invitent les enseignants d'EPS à réagir de manière plus éducative aux comportements perturbateurs de leurs élèves. D'abord, dans un sport à connotation masculine, les filles ne doivent pas avoir l'impression d'être visé personnellement par l'enseignant selon Merle (2005), le caractère unique d'une humiliation peut rompre définitivement le lien avec l'enseignant. De plus chaque faute doit être traitée de façon objective comme un 
événement isolé et il ne faut pas généraliser la sanction. En effet, les enseignants doivent admettre que leurs interventions, bien qu'elles soient fondées sur des intentions pédagogiques louables, peuvent parfois avoir des effets réels non désirés et surtout, défavorables au développement de leurs élèves. Alors ils doivent davantage s'interroger sur les effets que produisent leurs actions bien plus que sur la légitimité de celles-ci. À ce propos, les enseignants d'EPS doivent s'assurer que les règles de conduite sont préalablement connues des élèves et qu'elles seront utilisées uniquement pour mettre en place un climat favorable d'apprentissage et non des entraves à la liberté des élèves. Dans cette perspective, l'enseignant doit instaurer, en collaboration avec ses élèves, un système de règles de conduite et de sanctions qu'il peut appliquer de façon consistante et rationnelle auprès de tous.

\section{Conclusion}

En guise de conclusion, nous pensons que ces révélations témoignent de la nécessité et la pertinence de les interroger sur les conditions d'apprentissage que les élèves vivent dans leurs séance d'éducation physique. Leurs révélations ont mis en lumière des actions adoptées par les enseignants qui méritent d'être reconsidérées afin d'offrir des conditions d'apprentissage plus équitables. Dans cette perspective, les enseignants d'EPS doivent reconnaître qu'il existe souvent des multiples sources d'injustices inhérentes à l'enseignement de l'éducation physique et que la souffrance des élèves filles ou garçons face à ces injustices échappe souvent à leur vigilance. Nous estimons que l'intervention de l'enseignant et le type de relation qu'il entreprend avec ses élèves représente un facteur incontournable pour la bonne démarche de l'enseignement et pour la conservation d'un climat positif favorable pour l'apprentissage. A ce niveau, (Siedentop, 1991) parle de système de taches qui forment «l'écologie de la classe » et qui met en valeur: le système de taches d'apprentissage, le système d'organisation et le système des interactions sociales entre les acteurs de la relation éducative.

En effet, pour préserver un bon climat de la classe et gérer une vie positive sans incidents et sans comportements déviants et de sentiments d'injustice perçus par les élèves, l'enseignant doit recourir à des stratégies éducatives pour bien gérer sa discipline enseignée. Il doit entre autre créer un climat d'apprentissage basé sur le respect mutuel entre les enseignants et ses élevés. Par ailleurs imposer des règles semble être la meilleure stratégie pour préserver une bonne relation éducative. Ces règles recouvrent une fonction sociale intégrant des codes qui régissent les rapports entre les personnes et qui permettent de mettre en valeur les droits et les devoirs de chacun.

Enfin, nous pensons qu'il est indispensable pour l'enseignant de se référer aux perceptions des élèves et de les considérer comme une source d'informations au service de la régulation de l'enseignement.

On peut dire donc que la relation pédagogique ne fonctionne pas en «vase clos» et ne prend pas en considération seulement la relation triangulaire enseignant-enseigné et savoir, mais englobe aussi des concepts cachés qui peuvent intervenir dans la communication pédagogique et qui peuvent nous renseigner sur les multiples facettes de la relation éducative .Ces concepts sont les perceptions, les sentiments et les représentations des élèves qui peuvent être des sources explicatives possible de conduites et de comportement de ces derniers.

\section{Références}

[1]. Artaud, G. (1989). L'intervention éducative. Ottawa : Les Presses de l'Université d'Ottawa.

[2]. Bègue, F. (2009). Justice et cognition. In M. Duru-Bellat \& D. Meuret (Eds.), Les sentiments de justice à et sur l'école. (pp. 15-30). Bruxelles : De Boeck

[3]. Brown, J. P. (1979). Description of dyadic student-teacher interaction in the physical education activity cl a s s. Thèse de doctorat en science de l'exercise et du sport, University of North Carolina, Greensboro.

[4]. Caillet, V. (2009). L'argumentation de justice chez les élèves : les jugements de justice en situation. In M. Duru-Bellat \& D. Meuret (Eds.), Les sentiments de justice à et sur l'école (pp. 201-212). Bruxelles : De Boeck.

[5]. Combaz, G. (1992). Sociologie de l'éducation physique. Evaluation et inégalités de réussite.

[6]. Paris : PUF.

[7]. Couchot-Schiex, S. \& Trottin, B. (2005). Interactions enseignants/élèves en EPS : variations en fonction du sexe et du genre. In G. Cogérino (dir.), Filles et Garçons en EPS (pp.163-179). Paris : Editions Revue EPS

[8]. Cleuziou, J-P. (2000). L'analyse des menus et des notes dans "L'éducation physique et sportive : certification au Baccalauréat» (sous la direction de Bernard David) Documents et travaux de recherche en éducation $\mathrm{N}^{\circ} 39 \mathrm{Ed}$. INRP

[9]. Cleuziou, J-P. (2000). L'analyse des menus et des notes dans "L'éducation physique et sportive : certification au Baccalauréat» (sous la direction de Bernard David) Documents et travaux de recherche en éducation $\mathrm{N}^{\circ} 39 \mathrm{Ed}$. INRP

[10]. Debarbieux, (1990). La violence dans la classe. Paris : ESF.

[11]. Debarbieux, E. (1999). Désigner et punir. Remarques sur une construction ethnicisante au collège. In D. Meuret (Ed.), La justice du système éducatif (pp.194-212). Bruxelles : De Boeck.

[12]. Desvignes, S. \& Meuret, D. (2009). Les sentiments de justice des élèves en France et pourquoi. In M. Duru-Bellat \& D. Meuret (Eds.), Les sentiments de justice à et sur l'école (pp. 187-199). Bruxelles : De Boeck.

[13]. Dostie (1996). Analyse d'incidents disciplinaires vécus par des éducateurs physiques au primaire. Mémoire de maîtrise en sciences de l'activité physique. Département des sciences de l'activité physique, Université du Québec à Trois-Rivières.

[14]. Dubet, F. (1999). Sentiments et jugements de justice dans l'expérience scolaire. In D. Meuret (Ed.), La justice du système éducatif (pp.177-194). Bruxelles : De Boeck.

[15]. Dubet, F. (2004). L'école des chances. Qu'est-ce qu'une école juste? Paris : Seuil. 
[16]. Dyson, B.P. (1995). Students' voices in alternative elementary physical education programs. Journal of Teaching in Physical Education, 14(4), 394-407.

[17]. Gagnon, J., Martel, D., Brunelle, J.P., Tousignant, M. et Spallanzani, C. (2006). Les injustices perçues par les élèves: un message révélateur pour les enseignants. Cahiers de la recherche en éducation, 9(1), 3-20

[18]. Martel, D., Gagnon, J., Pelletier-Murphy, J. et Grenier, J. (1999). Pygmalion en éducation physique : un mythe bien réel. Revue canadienne de l'éducation, 24(1), 42-56.

[19]. Martinek, T.J. (1988). Confirmation of a teacher expectancy mode 1 : Student perceptions and causal attributions of teaching behaviors. Research Quarterly for Exercise and Sport, 59(2), 118-126.

[20]. Martinek, T.J. et Karper, W.B. (1986). Motor ability and instructional contexts: Effects on teacher expectations and dyadic interactions in elementary physical education classes. Journal of Classroom Interaction, 21(2), 16-25.

[21]. Martinek, T. J. (1989). The psycho-social dynamics of the Pygmalion phenomenon in physical education and sport.In T.J. Templin et P.G. Schempp (dir.), Learning to teach: Socialisation into physical education (p. 199-217). Indianapolis, IN: Benchmark Press Inc.

[22]. Merle, P. (1999). Equité et notation: l'expérience subjective des lycéens. In D. Meuret (Ed.). La justice du système éducatif (pp. 213-226). Bruxelles : De Boeck.

[23]. Merle, P. (2005). L'élève humilié. L'école, un espace de non-droit ? Paris : P.U.F.

[24]. Meuret, D. (1999). La justice du système éducatif. Bruxelles : De Boeck.

[25]. L'Écuyer, R. (1988). L'analyse de contenu: notion et étapes. In J.P. Deslauriers (dir.), Les méthodes de la recherche qualitative (p. 49-65). Québec: Presses de l’Université du Québec.

[26]. Rail, G. et Dallaire, H. (1997). La problématique de l'équité : parole aux enseignantes et enseignants en éducation physique. Revue Avante, 3(1), 34-47.

[27]. Rayou, P. (1999). "Combien t'as eu ? Les lycéens à l'épreuve de la dissertation de philosophie", In Denis Meuret (dir.), La justice du système éducatif, Bruxelles, De Bœck

[28]. Scraton, S. (1992). Shaping up to womanhood: Gender and girls'physical education. Buckingham: Open University Press.

[29]. Siedentop, D. (1991). Developing Teaching Skills in Physical Education - Third Edition. Palo Alto, CA: Mayfield.

[30]. Vanoutrive, J., Friant, N. \& Derobertmasure, A. (2011. L’injustice scolaire : quels sentiments chez les élèves ? ÉducationFormation, e-295, 129-139. En ligne : http://ute3.umh.ac.be/revues/ (page consultée le 23 septembre 2011).

[31]. Vigneron, C. (2005). Les écarts de réussite en EPS aux examens entre filles et garçons. In G. Cogérino (dir.), Filles et garçons en EPS (pp. 61-99). Paris : Edition Revue EPS, Collection « Recherche et formation ». 